\title{
Cats and Illusory Motion
}

\author{
Rasmus Bååth1, Takeharu Seno 2* ${ }^{*}$ Akiyoshi Kitaoka ${ }^{3}$ \\ ${ }^{1}$ Cognitive Science, Department of Philosophy, Lund University, Lund, Sweden \\ ${ }^{2}$ Institute for Advanced Study, Faculty of Design, Research Center for Applied Perceptual Science, \\ Kyushu University, Fukuoka, Japan \\ ${ }^{3}$ Department of Psychology, Ritsumeikan University, Kyoto, Japan \\ Email: rasmus.baath@lucs.lu.se, ${ }^{*}$ seno@design.kyushu-u.ac.jp, akitaoka@lt.ritsumei.ac.jp
}

Received 29 April 2014; revised 26 May 2014; accepted 21 June 2014

Copyright (C) 2014 by authors and Scientific Research Publishing Inc.

This work is licensed under the Creative Commons Attribution International License (CC BY).

http://creativecommons.org/licenses/by/4.0/

c) (i) Open Access

\begin{abstract}
We present the first evidence that cats experience visual illusions and that a non-human animal can see illusory motion. In three videos we show cats reacting with hunting behavior when watching the Rotating Snakes illusion. This is taken to mean that cats see illusory motion in this image due to the propensity of cats to pursue movement. This is further supported by a survey where $29 \%$ of the respondents answered that their cat reacted to the illusion. A number of preferential looking experiments were also indicative of cats experiencing the illusion, but not conclusively so.
\end{abstract}

\section{Keywords}

\section{Cat, Illusory Motion, Rotating Snakes Illusion, Cat Vision, Visual Illusions}

\section{Introduction}

Many cat owners have probably looked into their cat's eyes and wondered how the world looks from its perspective. While there might never be a satisfactory answer to that question a video, published by the first author on YouTube ${ }^{1}$ last year (Figure 1), at least indicated one aspect in which cats and humans perceive the world in a similar way. In that movie a young cat is shown a paper with the Rotating Snakes illusion, a static image that nevertheless induces a strong sense of motion, and reacts like most young cats do when they see a moving object: by chasing it. The Rotating Snakes illusion (shown in Figure 2) was discovered by Kitaoka (2003) and it depends on the local arrangement of four color regions of different luminance: black (darkest), blue (second darkest), white (lightest), yellow (second lightest) (Kitaoka \& Ashida, 2003; Kuriki et al., 2008). This illusion is called the Rotating Snakes illusion and can be considered an optimized version of either the Fraser-Wilcox illusion

*Corresponding author.

1'http://youtu.be/CcXXQ6GCUb8. 


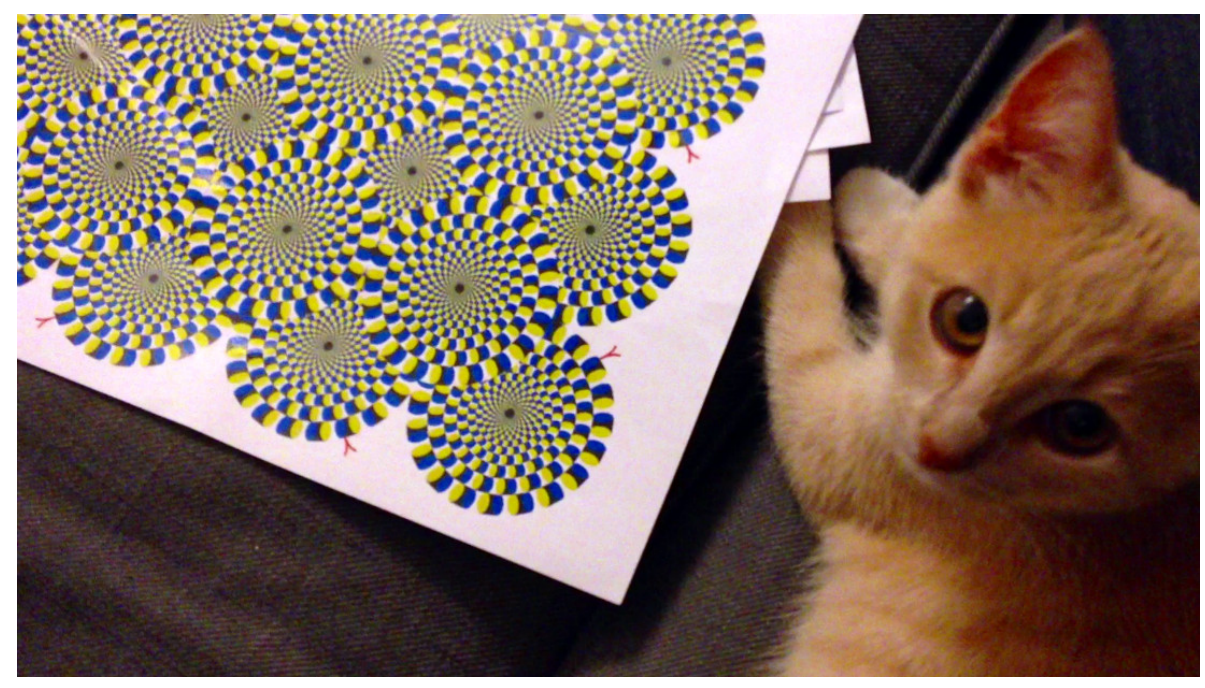

Figure 1. A frame from the first video showing a cat reacting to the Rotating Snakes illusion.
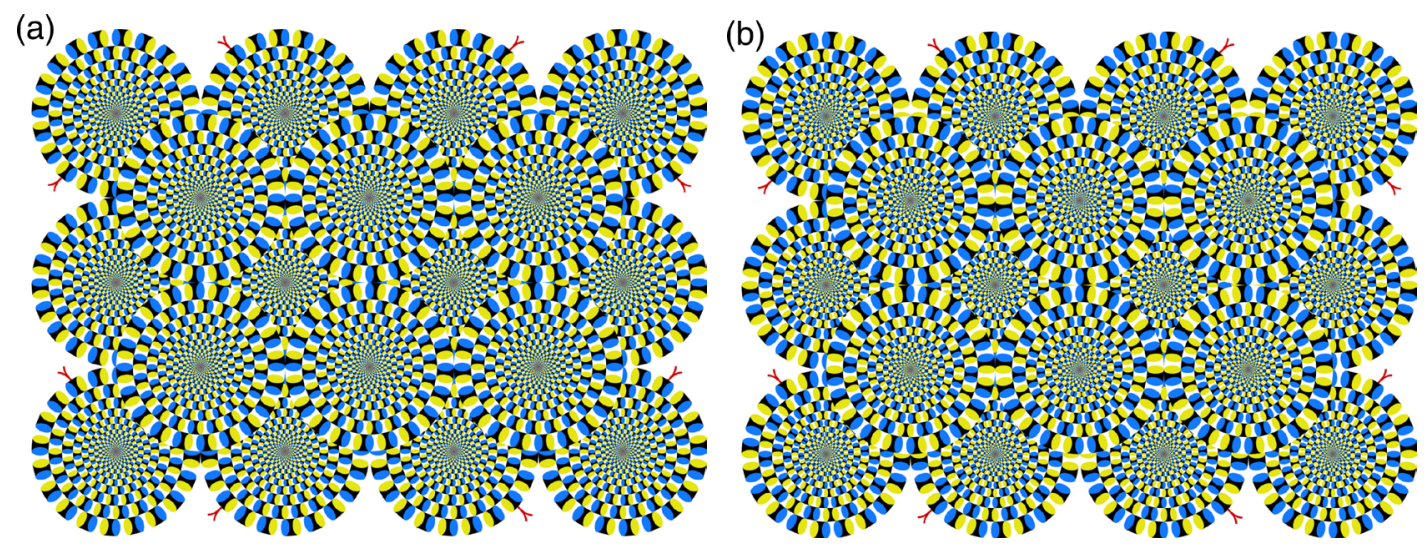

Figure 2. (a) The Rotating Snakes illusion and (b) the control image.

(Fraser \& Wilcox, 1979; Naor-Raz \& Sekuler, 2000) or the peripheral drift illusion (Faubert \& Herbert, 1999). Conway et al. (2005) suggested that these responses occur in the neurons in MT (middle temporal) and Kuriki et al. (2008) showed that in humans hMT+ (middle temporal complex) responds to this illusion. Moreover, Ashida et al. (2012) showed evidence that V1 (primary visual cortex) - V4, V3A (V3 accessory), and MT+ are involved in this illusion. Recently, Kanazawa et al. (2013) reported that six- to eight-month-old infants see the illusory motion in the Rotating Snakes illusion, however, it has never been shown that non-human species experiences this illusion.

The movie of the cat reacting to the Rotating Snakes illusion was then novel in two ways: 1) It is the first example of a cat reacting to a visual illusion and 2) it is the first example of an animal reacting to illusory motion. The video was soon followed by two more showing cats reacting to the Rotating Snakes illusion by chasing the illusory motion ${ }^{2}$. As convincing as they are, these videos are strictly only anecdotal evidence that cats see the Rotating Snakes illusion. We therefore made two attempts at gathering data to back up this proposition.

\section{Experimental Data}

An online survey was conducted where the survey participants were asked to show the Rotating Snakes illusion to their pet cats and report whether their cat reacted to the illusion, in what way it reacted and how old their cat was. We included the age question as we hypothesized that young cats would be more likely to react to the illusory motion as they tend to respond to motion to a higher degree than older cats (West, 1974). Participants were

${ }^{2}$ http://youtu.be/kcSpuMR_PRE and http://youtu.be/1KrboUI1hg8. 
recruited by putting an advertisement in the original video showing a cat that reacts to the illusion. Out of the 66 respondents 19 answered that their cat responded to the illusion. The reaction of one of these cats was described as "[it] attacked the rotating illusions. Seemed to attack one point, then notice another moving illusion [and] then attack that" and another by "She seemed interested in it, moving her sight from circle to circle". These reports, describing the cats shifting their attention between the circles, is in agreement with that the illusory motion perception occurs mainly in the peripheral visual field (Kuriki et al., 2008). As hypothesized the cats that responded to the illusion were on average 2.4 years younger than the cats that did not react (two sample t-test, $p=0.035, t$ $=-2.21)$.

Encouraged by these results we performed a preferential looking experiment, an experimental paradigm first used to investigate infant's vision (Fantz, 1963) but recently also used in animal experiments (Shirai et al., 2010; Tomonaga et al., 2004; Shirai \& Imura, 2014). This experiment was conducted in a "cat-café" in Fukuoka-city. It should be noted that this was not a café for cats but rather for humans that would like to have coffee while interacting with the many cats that live in the café. The Rotating Snakes illusion and its control image were printed on high luminance photo-paper and fixed to the floor (see Figure 2) where eleven cats were allowed to freely explore the images. Four trials were filmed ${ }^{3}$, each trial lasted 10 minutes and the position of the illusion and control image were switched between each trial. Five naïve persons assessed the amount of time the cats visited each of the images and their assessments were averaged. Out of the total 40 minutes the cats spent $816 \mathrm{~s}$ visiting the illusion and $390 \mathrm{~s}$ visiting the control image. That is, the cats spent more than twice the amount of time visiting the Rotating Snakes illusion. However, we did not observe the same hunting behavior as seen in the original three videos and in general the cats showed little interest in any of the images. We conducted four similar experiments, one on the same group of cats and three on other groups of domestic cats, without being able to invoke any hunting behavior, rather the cats paid little attention to both the illusion and control images. A litter of five eight week old kittens were also shown the illusion but failed to pay any attention.

\section{Discussion}

There is a growing literature regarding animals and visual illusions. There have been the reports of that chimpanzees experience visual illusions such as the illusion of brightness (Gunter, 1954), the Ponzo illusion (Fujita, 1997), the Müller-Lyer illusion (Suganuma et al., 2007), and the corridor illusion (Imura \& Tomonaga, 2009). There is also evidence that pigeons experience visual illusions. Nakamura et al. $(2006,2008)$ reported that the Müller-Lyer illusion and the Ebbinghaus-Titchener illusion are experienced by pigeons. Given the videos published recently and the result of the survey we would like to add cats to the list of animals that experience visual illusions. If you, as a reader of Psychology, would like to test whether your cat responds to the Rotating Snakes illusion both the illusion and the control images shown in Figure 1 are available for download at http://www.psy.ritsumei.ac.jp/ akitaoka/rotsnakes-test-control.html.

\section{Acknowledgements}

This work is supported by the Program to Disseminate Tenure Tracking System, MEXT, Japan and by Swedish Research Council grant 349-2007-8695. We thank Cat-Café “Ailuros” for providing us 11 cats. We also thank Honami Yamashita, Junya Matsufuji, Ayaka Kubokawa, Yuka Egashira and Takayuki Matsuoka for their analyses of the movies. At last we thank Stina, the first cat to be filmed playing with the Rotating Snake Illusion, and her owner Maria Lennartsson.

\section{References}

Ashida, H., Kuriki, I., Murakami, I, Hisakata, R., \& Kitaoka, A. (2012). Direction-Specific fMRI Adaptation Reveals the Visual Cortical Network Underlying the “Rotating Snakes” Illusion. NeuroImage, 61, 1143-1152. http://dx.doi.org/10.1016/j.neuroimage.2012.03.033

Conway, B. R., Kitaoka, A., Yazdanbakhsh, A., Pack, C. C., \& Livingstone, M. S. (2005). Neural Basis for a Powerful Static Motion Illusion. Journal of Neuroscience, 25, 5651-5656. http://dx.doi.org/10.1523/JNEUROSCI.1084-05.2005

Fantz, R. L. (1963). Pattern Vision in Newborn Infants. Science, 140, 296-297.

http://dx.doi.org/10.1126/science.140.3564.296

${ }^{3}$ The movies are available at http://www.youtube.com/user/smXaki. 
Faubert, J., \& Herbert, A. M. (1999). The Peripheral Drift Illusion: A Motion Illusion in the Visual Periphery. Perception, 28, 617-621. http://dx.doi.org/10.1068/p2825

Fraser, A., \& Wilcox, K. J. (1979). Perception of Illusory Movement. Nature, 281, 565-566. http://dx.doi.org/10.1038/281565a0

Fujita, K. (1997). Perception of the Ponzo Illusion by Rhesus Monkeys, Chimpanzees, and Humans: Similarity and Difference in the Three Primate Species. Perception \& Psychophysics, 59, 284-292. http://dx.doi.org/10.3758/BF03211896

Gunter, R. (1954). The Discrimination between Lights of Different Wavelengths in Cat. Journal of Comparative and Physiological Psychology, 47, 169-172. http://dx.doi.org/10.1037/h0061325

Imura, T., \& Tomonaga, M. (2009). Moving Shadows Contribute to the Corridor Illusion in a Chimpanzee (Pan troglodytes). Journal of Comparative Psychology, 123, 280-286. http://dx.doi.org/10.1037/a0015839

Kanazawa, S., Kitaoka, A., \& Yamaguchi, M. K. (2013). Infants See Illusory Motion in Static Figures. Perception, 42, 828-834. http://dx.doi.org/10.1068/p7460

Kitaoka, A. (2003). Rotating Snakes. http://www.ritsumei.ac.jp/ akitaoka/index-e.html

Kitaoka, A., \& Ashida, H. (2003). Phenomenal Characteristics of the Peripheral Drift Illusion. Vision, 15, 261-262.

Kuriki, I., Ashida, H., Murakami, I., \& Kitaoka, A. (2008). Functional Brain Imaging of the Rotating Snakes Illusion by fMRI. Journal of Vision, 8, 16.1-10.

Nakamura, N., Fujita, K., Ushitani, T., \& Hiromitsu, M. (2006). Perception of the Standard and the Reversed Müller-Lyer Figures in Pigeons (Columba livia) and Humans (Homo sapiens). Journal of Comparative Psychology, 120, $252-261$. http://dx.doi.org/10.1037/0735-7036.120.3.252

Nakamura, N., Watanabe, S., \& Fujita, K. (2008). Pigeons Perceive the Ebbinghaus-Titchener Circles as an Assimilation Illusion. Journal of Experimental Psychology: Animal Behavior Processes, 34, 375-387.

http://dx.doi.org/10.1037/0097-7403.34.3.375

Naor-Raz, G., \& Sekuler, R. (2000). Perceptual Dimorphism in Visual Motion from Stationary Patterns. Perception, 29, 325335.

Shirai, N., \& Imura, T. (2014). Looking Away before Moving Forward: Changes in Optic Flow Perception Precede Locomotor Development. Psychological Science, 25, 485-493. http://dx.doi.org/10.1177/0956797613510723

Shirai, N., Imura, T., Hattori, Y., Adachi, I., Ichihara, S., Kanazawa, S., Yamaguchi, M. K., \& Tomonaga, M. (2010). Asymmetric Perception of Radial Expansion Contraction in Japanese Macaque Infants. Experimental Brain Research, 202, 319-325. http://dx.doi.org/10.1007/s00221-009-2136-3

Suganuma, E., Pessoa, V. F., Monge-Fuentes, V., Castro, B. M., \& Tavares, M. C. (2007). Perception of the Müller-Lyer Illusion in Capuchin Monkeys (Cebus apella). Behavioral Brain Research, 182, 67-72. http://dx.doi.org/10.1016/j.bbr.2007.05.014

Tomonaga, M., Tanaka, M., \& Matsuzawa, T. (2004). Development of Social Cognition in Infant Chimpanzees (Pan troglodytes): Face Recognition, Smiling, Gaze, and the Lack of Triadic Interactions. Japanese Psychological Research, 46, 227235. http://dx.doi.org/10.1111/j.1468-5584.2004.00254.x

West, M. (1974). Social Play in the Domestic Cat. American Zoologist, 14, 427-436. 
Scientific Research Publishing (SCIRP) is one of the largest Open Access journal publishers. It is currently publishing more than 200 open access, online, peer-reviewed journals covering a wide range of academic disciplines. SCIRP serves the worldwide academic communities and contributes to the progress and application of science with its publication.

Other selected journals from SCIRP are listed as below. Submit your manuscript to us via either submit@scirp.org or Online Submission Portal.
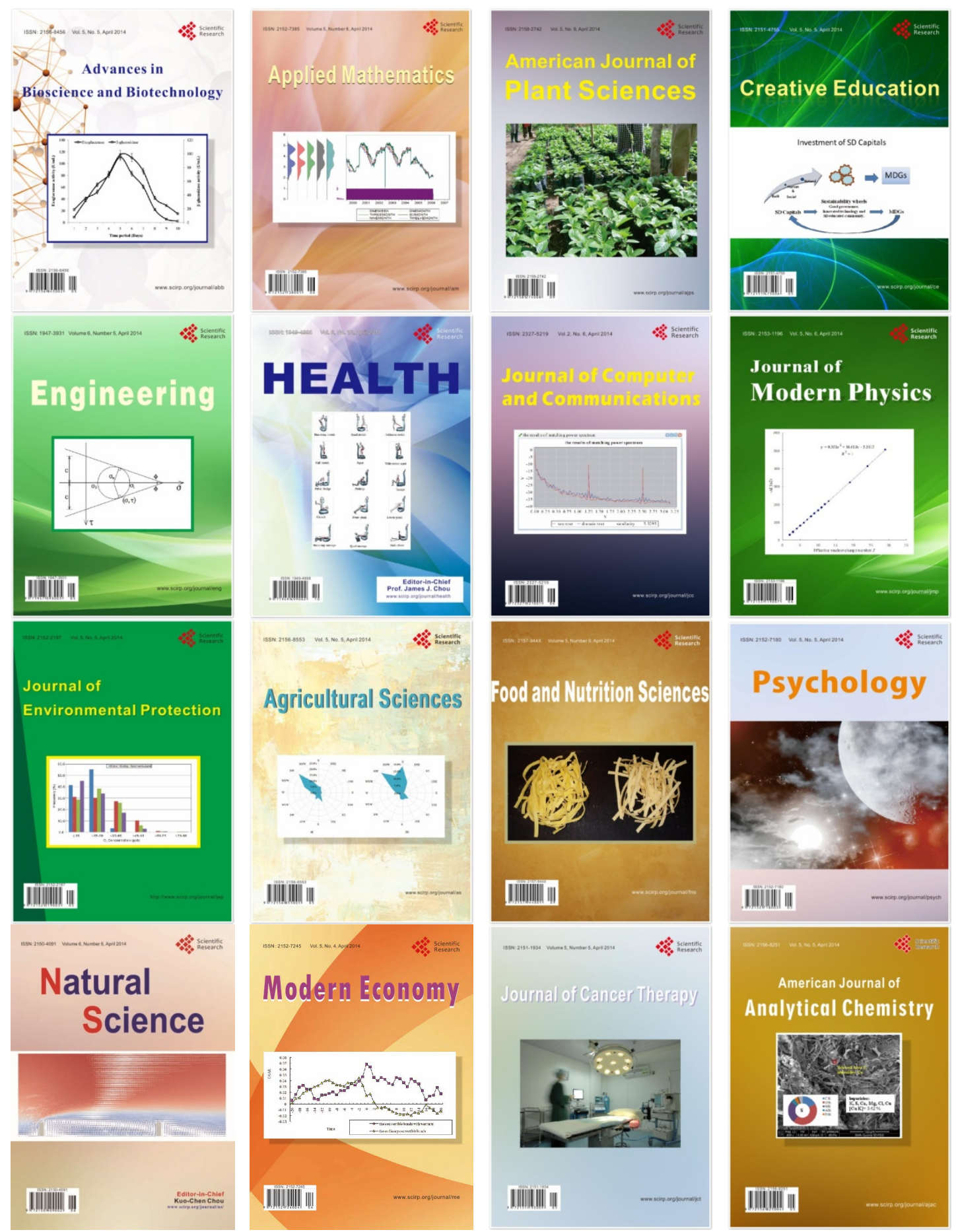